\title{
CLAVE PARA LA IDENTIFICACIÓN DE GÉNEROS DE THRIPS (INSECTA: THYSANOPTERA) COMÚNMENTE ASOCIADOS A PLANTAS ORNAMENTALES EN CENTROAMÉRICA
}

\author{
KEY TO THE IDENTIFICATION OF GENERA OF THRIPS (INSECTA: THYSANOPTERA) \\ COMMONLY ASSOCIATED WITH ORNAMENTAL PLANTS IN CENTRAL AMERICA
}

\section{Gerardo A. SOTO-RODRÍGUEZ, ${ }^{1, *}$ JeSÚS AleXANDER RODRÍGUEZ-ARRIETA, ${ }^{1,2,3}$ CARLOS GONZÁLEZ MUÑOZ,${ }^{4}{ }^{\text {JHONATHAN CAMBERO-CAMPOS }}{ }^{5}$ y AXEL P. RETANA-SALAZAR ${ }^{1,6}$}

\author{
${ }^{1}$ Centro de Investigación en Estructuras Microscópicas (CIEMIC), Ciudad de la Investigación, Universidad de \\ Costa Rica 2060. \\ ${ }^{2}$ Escuela de Biología, Ciudad Universitaria Rodrigo Facio, Universidad de Costa Rica 2060. \\ ${ }^{3}$ Escuela de Ciencias Agrarias, Universidad Nacional de Costa Rica, Heredia, Costa Rica. \\ ${ }^{4}$ Facultad de Agronomía, Universidad Agraria de la Habana, Cuba. \\ ${ }^{5}$ Escuela de Agronomía, Universidad Autónoma de Nayarit (UAN), Nayarit, México. \\ ${ }^{6}$ Escuela de Nutrición, Facultad de Medicina, Ciudad de la Investigación, Universidad de Costa Rica 2060. \\ * Autor para correspondencia:<cultivosyasesorias@yahoo.com>.
}

Recibido: 06/10/2016; aceptado: 10/08/2017.

Editor responsable: Pedro Reyes Castillo.

Soto-Rodríguez, G. A., Rodríguez-Arrieta, J. A., González Muñoz, C., Cambero-Campos, J. y Retana-Salazar, A. P. (2017) Clave para la identificación de géneros de thrips (Insecta: Thysanoptera) comúnmente asociados a plantas ornamentales en Centroamérica. Acta Zoológica Mexicana (n.s), 33(3), 454-463.

RESUMEN. Se presenta una clave ilustrada de especies de tisanópteros encontradas en plantas ornamentales de Costa Rica, Cuba y México, con referencias de especies presentes en la Argentina y la Península Ibérica. La clave incluye un total de 21 especies, de las cuales 16 pertenecen al suborden Terebrantia y cinco al suborden Tubulifera. Para todas las especies se indican los sitios de recolección y la planta hospedadora correspondiente.

Palabras clave: Thysanoptera, Terebrantia, Tubulifera, clave de identificación, cultivos ornamentales.

\section{INTRODUCCIÓN}

La producción de plantas ornamentales ha tomado mucho auge a nivel mundial, convirtiéndose en una actividad económica muy lucrativa, con cadenas de producción donde las principales empresas productoras y compradoras de plantas ornamentales se ubican en Europa. La Unión Europea cuenta con un 12\% del área total de producción (European Commission Directorate-General For Agriculture And Rural Development, 2006). Alemania en el 2008 tuvo ventas por 1500 millones de euros en plantas de exteriores (ornato) y 270 millones en plantas de inte-
Soto-Rodríguez, G. A., Rodríguez-Arrieta, J. A., González Muñoz, C., Cambero-Campos, J., \& Retana-Salazar, A. P. (2017) Key to the identification of genera of thrips (Insecta: Thysanoptera) commonly associated with ornamental plants in Central America. Acta Zoológica Mexicana (n.s), 33(3), 454-463.

ABSTRACT. An illustrated key for the species of Thysanoptera in ornamental crops in Central America is presented. The key includes a total of 21 species, distributed in 16 in the suborder Terebrantia and five in the suborder Tubulifera. In all cases the location of collections is indicated and when if possible also the ornamental crop which was found.

Key words: Thysanoptera, Terebrantia, Tubulifera, identification key, ornamental crops.

riores (Federal Ministry of Food, Agriculture and Consumer Protection, 2010).

En 2005 un estudio de la Universidad de Florida establece que el sector de producción de plantas ornamentales estimó un desarrollo en las ventas de 15,24 US\$ billones (Hodges \& Haydu, 2006). En estos casos los productores mayoristas llegan a tener ganancias de más de 3US\$ billones (Mizell et al., 2010).

Entre los países productores de América Latina que abastecen a Europa de esta clase de cultivos se hallan México, Cuba, Colombia, Costa Rica, principalmente. Estos países se caracterizan por una diversidad amplia de 
productos cultivados y al mismo tiempo por sus conexiones tropicales de alta vulnerabilidad en cuanto a infestación de plagas en cultivos de esta índole.

Uno de los grupos de insectos de gran importancia por sus efectos como plaga directa o indirecta, por la trasmisión de virus e inclusive por su potencial como biocontroladores de otras plagas, son los tisanópteros. Por ello, el conocimiento de la diversidad de especies de tisanópteros es necesario para establecer normas de control fitosanitario para este tipo de cultivos, que requieren anualmente de altas inversiones, tanto por las empresas productoras como por los mismos estados que buscan minimizar la entrada de plagas en sus importaciones.

La detección de estos insectos diminutos se realiza de forma visual a nivel de los estados adulto y larva. Debido a la dificultad de ver las formas inmaduras a simple vista éstas pueden pasar desapercibidas a los controles visuales. A esto se suma la dificultad de identificar especies sin que éstas hayan desarrollado enteramente las características de un adulto (Jagadish \& Ananthakrishnan, 1972).

El proceso normal de inspección es que al ser detectada una larva o pupa de tisanóptero el producto es devuelto considerándose que la planta no está libre de plagas. En la legislación de algunos países de América Latina se contempla la destrucción delante del inspector correspondiente cuando se detecta alguna plaga cuarentenaria en un determinado cargamento (PCFOE, 2008). El primer paso para reconocer los estados inmaduros de tisanópteros presentes en cultivos ornamentales es contar con la correcta identificación de los adultos de las especies plaga para lo cual es necesario generar claves específicas y establecer las correspondientes asociaciones con las plantas ornamentales.

\section{MATERIALES Y MÉTODOS}

Para el desarrollo de este trabajo se contó con la revisión del material de las colecciones de Thysanoptera de la Universidad de Costa Rica, la colección del Instituto de Biología, Universidad Nacional Autónoma de México (IBUNAM), la colección de la Universidad Autónoma de Nayarit (UAN) como material del sistema de Sanidad Vegetal de Cuba y de la colección de Thysanoptera de la Facultad de Agronomía de la Universidad Agraria de la Habana. Además, se efectuó la revisión de material bibliográfico de informes para América Central de especies de thrips asociadas a ornamentales.

\section{RESULTADOS}

A continuación, se presenta una lista de los géneros de Thysanoptera y plantas asociadas, registrados en Costa Rica y la mayor parte de América Central (Cuadro 1) y una clave ilustrada para la identificación de los mismos. Se incluyen, además, aquellos citados para Cuba y México, con referencias de thrips asociados a sistemas de cultivos de ornamentales en la Península Ibérica y la Argentina.

\section{Clave para la identificación de los géneros de Thysanoptera asociados a la producción de plantas ornamentales}

1a) Tergo abdominal $X$ en forma de tubo (Fig. 1A); alas sin microtriquias ni venas longitudinales (Fig. 1B, C); ovipositor membranoso........... Tubulifera 2 1b) Tergo abdominal $X$ termina en punta cónica (Fig. 2A); alas con microtriquias y venas longitudinales (Fig. 2B, C); ovipositor generalmente bien desarrolla-

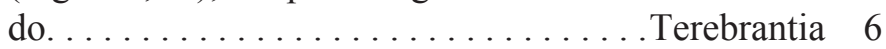
2a) Cabeza y pronoto fuertemente ornamentados o con tubérculos; número de segmentos antenales variable (Fig.

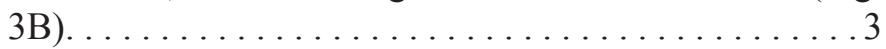
2b) Cabeza y pronoto lisos; disposición de los conos sen-

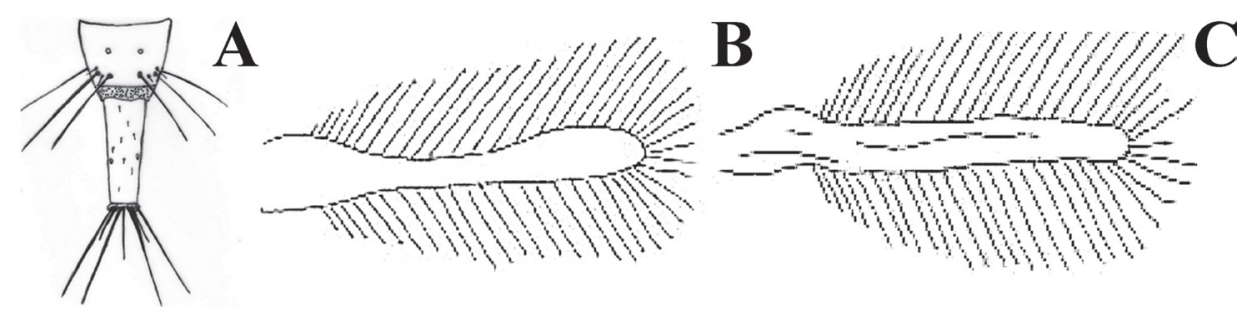

Figura 1. Tubulifera. A Tergo X (tubo) (redibujado de Palmer et al., 1989). B-C Alas de Phlaeothripidae, B: ala anterior de Karnyothrips. C: ala anterior de Liothrips (Tomadas de Soto-Rodríguez \& Retana-Salazar, 2003). 
Cuadro 1. Lista de especies de thrips registradas en Costa Rica y América Central en plantas de uso ornamental.

\begin{tabular}{|c|c|c|}
\hline Thysanoptera & Planta & Referencia \\
\hline $\begin{array}{l}\text { TUBULIFERA } \\
\text { Phlaeothripidae }\end{array}$ & $\begin{array}{l}\text { - Cattleya trianae Linden \& Reichb. } \\
\text { (Orchidaceae) }\end{array}$ & Suris \& González (2008) \\
\hline Stephanothrips occidentalis Hood \& Williams & - Hojarasca & Mound \& Marullo (1996) \\
\hline Gynaikothrips ficorum Marchal & Ficus microcarpa L. (Moraceae) & Mound \& Marullo (1996) \\
\hline Gynaikothrips uzeli Zimmermann & Ficus benjamina L. (Moraceae) & Mound \& Marullo (1996) \\
\hline Aleurodothrips fasciapennis Franklin & $\begin{array}{l}\text { - Cattleya trianae Linden \& Reichb. } \\
\text { (Orchidaceae) y Rosa indica L. (Rosaceae) } \\
\text { - Depredadora de cochinillas de escama y } \\
\text { mosca blanca }\end{array}$ & $\begin{array}{l}\text { Suris \& González (2008) } \\
\text { (Palmer \& Mound, 1991) }\end{array}$ \\
\hline Haplothrips graminis Hood & $\begin{array}{l}\text { En múltiples hospederos en invernaderos de } \\
\text { ornamentales }\end{array}$ & $\begin{array}{l}\text { única especie recolectada en ornamentales } \\
\text { en Costa Rica (Retana-Salazar et al., 2014) }\end{array}$ \\
\hline Hoplandrothrips sp. & $\begin{array}{l}\text { Zinnia elegans Jacq (Asteraceae); } \\
\text { Dianthus caryophyllus L. (Cariofilaceae); } \\
\text { Canna glauca L. (Cannaceae) }\end{array}$ & Suris \& González (2008) \\
\hline \multicolumn{3}{|l|}{$\begin{array}{l}\text { TEREBRANTIA } \\
\text { Heterothripidae }\end{array}$} \\
\hline Heterothrips sericatus Hood & En flores de Citrus y de Mimosa sp. & Mound \& Marullo (1996) \\
\hline Heterothrips lankesteriensis Retana-Salazar & Malpighia glabra L. (Malpighiaceae) & Retana-Salazar (2009) \\
\hline \multicolumn{3}{|l|}{ Thripidae } \\
\hline Caliothrips phaseoli Daniel & $\begin{array}{l}\text { Chrysanthemum coronarium } \mathrm{L} \text {. } \\
\text { (Amaranthaceae) }\end{array}$ & Suris \& González (2008) \\
\hline Partenothrips dracaenae Heeger & Dianella sp. (Xanthorrhoeaceae) & Gagné \& Montgomery (1985) \\
\hline Heliothrips haemorrhoidalis Bouché & $\begin{array}{l}\text { - En cítricos, helechos y ornamentales de } \\
\text { invernaderos } \\
\text { - Helecho } \\
\text { Phlebodium pseudoaureum (Cav.) Lellinger } \\
\text { bajo invernadero }\end{array}$ & A.P. Retana-Salazar, com. pers. \\
\hline Exophthalmothrips fulvipennis Moulton & $\begin{array}{l}\text { Megaskepasma (Acanthaceae) y en } \\
\text { invernaderos de Impatiens sp. (Balsaminaceae) }\end{array}$ & Retana-Salazar (1998) \\
\hline $\begin{array}{l}\text { Frankliniella occidentalis Pergande y } \\
\text { Frankliniella insularis Franklin }\end{array}$ & $\begin{array}{l}\text { Varias especies ornamentales y cerca de } 60 \\
\text { plantas hospederas }\end{array}$ & Retana-Salazar \& Rodríguez-Arrieta (2012) \\
\hline Gonzalezya marinae Retana-Salazar & Anthurium sp. & Retana-Salazar (2007) \\
\hline Microcephalothrips abdominalis Crawford & Helianthus annus L. (Asteraceae); & Mound \& Marullo (1996) \\
\hline Thrips orientalis Bagnall & Gardenia jasminoides Ellis (Rubiaceae) & Suris \& González, 2008 \\
\hline Thrips palmi Karny & $\begin{array}{l}\text { Amaranthus cruentus L. (Acanthaceae); } \\
\text { Dalia cuccinea Cav., Helianthus annus L. y } \\
\text { Zinnia elegans Jacq (Asteraceae); Dianthus } \\
\text { caryophyllus L. (Cariofilaceae); Manosa } \\
\text { alliaceae L. (Bignoniaceae); Plumbago } \\
\text { capensis Thub. (Plumbaginaceae); Rosa indica } \\
\text { (Rosaceae) }\end{array}$ & Suris \& González, 2008 \\
\hline Thrips florum Schmutz & Gardenia augusta L. (Rubiaceae) & Mound \& Marullo (1996) \\
\hline
\end{tabular}




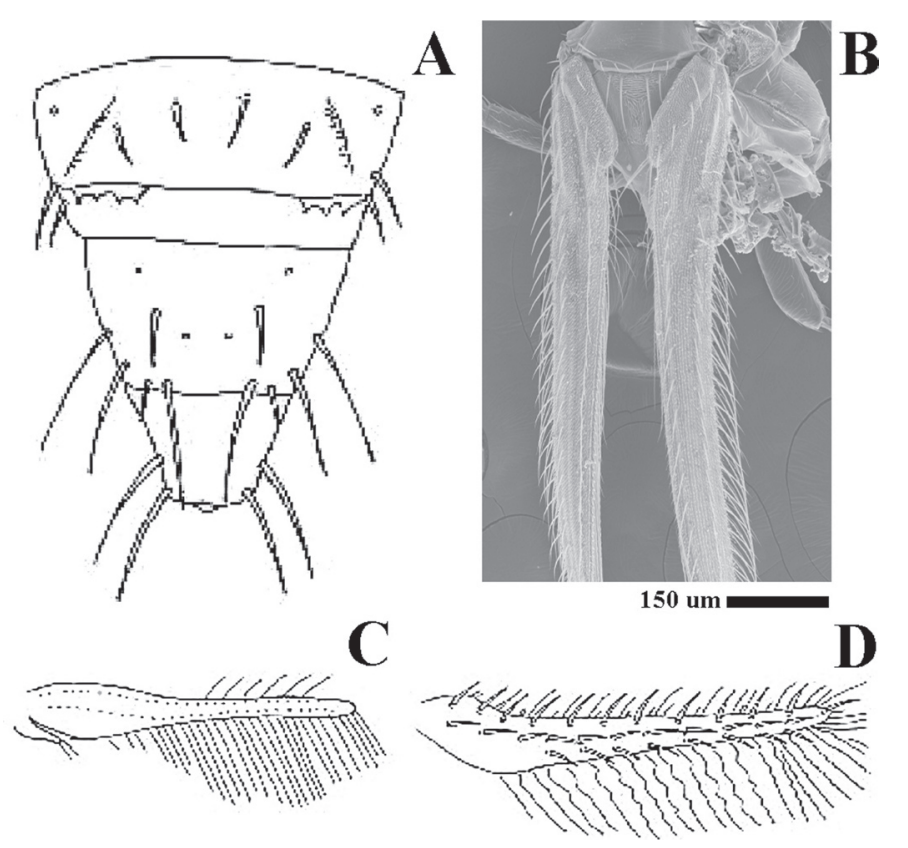

Figura 2. Terebrantia. A Sección terminal del abdomen (tergos VIIIX). Alas, B: alas anteriores de Frankliniella insularis (Franklin) $(2,1$ $\mathrm{cm}=300 \mu \mathrm{m}$ ), C: ala anterior de Heliothrips (Panchaetothripinae), D: ala anterior de Selenothrips (Panchaetothripinae) (tomadas de SotoRodríguez \& Retana-Salazar, 2003).

soriales en los antenómeros III-IV en número diferente a

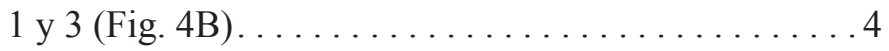
3a) Antena con ocho antenómeros (Fig. 3A), antenómero III con un cono sensorial y antenómero IV con tres conos sensoriales; sedas en el margen anterior de la cabeza ausentes.................... Gynaikothrips

Gynaikothrips ficorum (Marchal) afecta a Ficus microcarpa L. F. mientras que Gynaikothrips uzeli es habitual en Ficus benjamina L. Las hojas son los órganos donde se evidencia el ataque de estas especies, al producir agallas abiertas (Morris et al., 1999). Algunos autores consideran que este daño es solo cosmético y que no afecta a la planta en su desarrollo fisiológico, además consideran que la tasa de renovación del follaje contrarresta el efecto de los thrips (Áreas-Sevilla \& González-López, 2008). No obstante, estudios detallados de los efectos en las hojas indican que no solo la afectan a nivel de producir deformidad del órgano foliar, sino que transforma el tejido parenquimatoso en tejido de origen embrionario, produciendo un cambio fisiológico serio a la hoja como órgano, además se registran en estas hojas gran cantidad de hifas de hongos y otros organismos (Retana-Salazar \& Sánchez-Chacón, 2009). Esto indica que posiblemente la
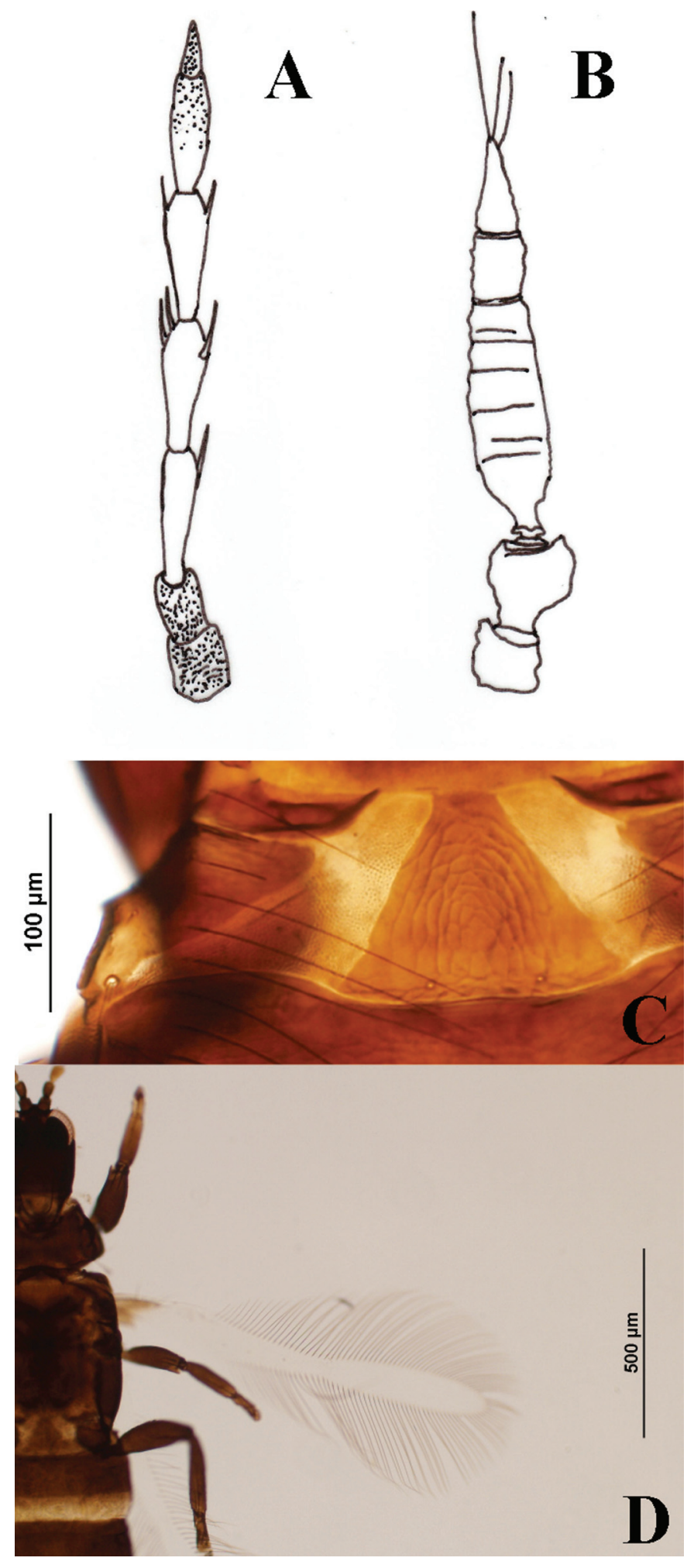

Figura 3. A: Antena de Gynaikothrips y Liothrips (tomada de Palmer et al. 1989). B: Antena de Stephanothrips (tomada de Mound \&

Marullo, 1996). C: Pelta completa de Haplothrips nigricornis. D: Ala anterior de Haplothrips nigricornis. 


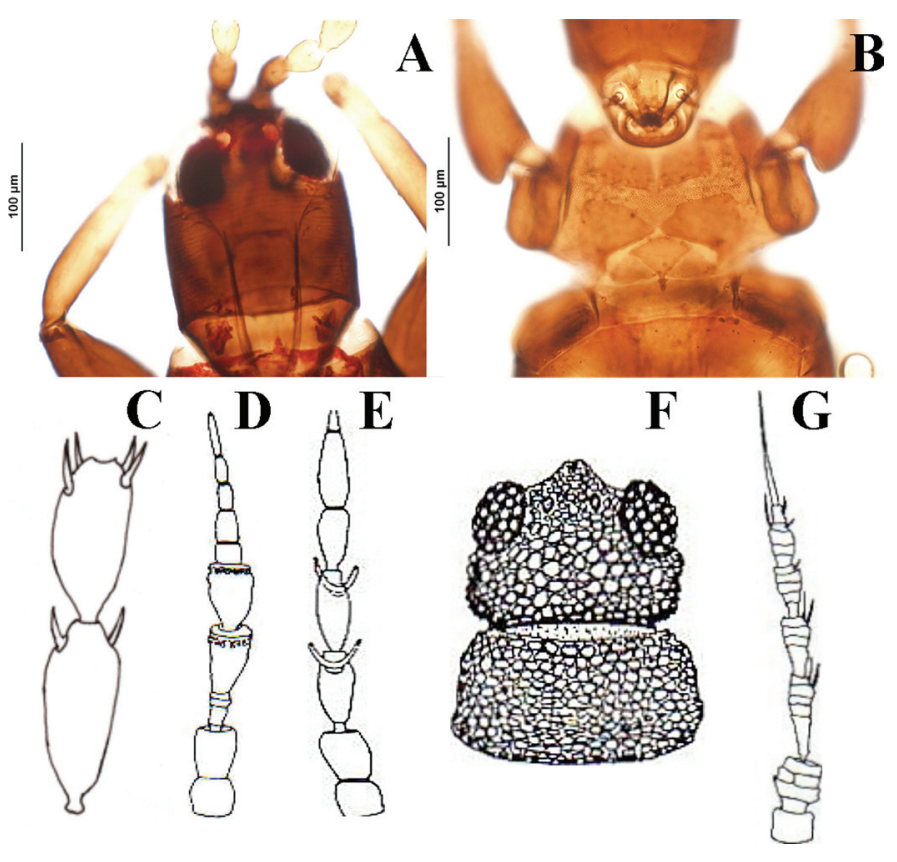

Figura 4. Haplothrips spp. A. H. gowdeyi. Cabeza vista dorsal mostrando los estiletes y el puente maxilar. B. H. reuteri. Pronoto vista ventral mostrando las placas del praepectus (basantra), placas inmediatamente debajo del cono bucal. C. Antenómeros III-IV de Haplothrips sp. (redibujados de Soto-Rodríguez \& Retana-Salazar, 2003). D. Antena de Heterothrips spp. (tomada de Mound et al.,

1993). E. Frankliniella spp. Antena (tomada de Mound et al., 1993). F. Heliothrips haemorrhoidalis (Bouché), ornamentación de la cabeza y el pronoto. G. Heliothrips haemorrhoidalis (Bouché), antena (tomadas de Mound et al., 1993).

hoja es más susceptible de ser afectada cuando se encuentra atacada por esta especie de thrips.

3b) Antena con cinco antenómeros, antenómeros III-V fusionados (Fig. 3B); cabeza cubierta de tubérculos, margen anterior de la cabeza con tres pares de sedas bien desarrolladas...................Stephanothrips

Stephanothrips occidentalis Hood \& Williams, se registró en Cuba asociada a Cattleya trianae Linden \& Reichb. (Orchidaceae) (Suris \& González, 2008). Hembras de esta especie fueron recolectadas de la hojarasca en Cuba y Costa Rica (Mound \& Marullo, 1996). Se trata de una especie micófaga frecuente en hojarasca seca.

4a) Cuerpo de color castaño oscuro; pelta completa (Fig. 3C); generalmente con sedas accesorias en las alas (Fig.

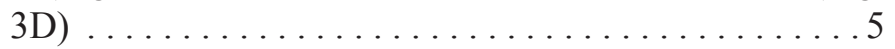
4b) Cuerpo bicoloreado; pelta dividida en dos escleritos posterolaterales $y$ un esclerito medio débilmente desarrollado; alas anteriores sin sedas accesorias....................... Aleurodothrips
Aleurodothrips fasciapennis Franklin, única especie recolectada en ornamentales en Cuba, en Cattleya trianae Linden \& Reichb. (Orchidaceae) y Rosa indica L. (Rosaceae) (Suris \& González, 2008). Depredadora de cochinillas de escama y mosca blanca (Palmer \& Mound, 1991) 5a) Puente maxilar bien desarrollado (Fig. 4A), estiletes maxilares separados por una distancia entre un cuarto o un tercio del ancho de la cabeza, cono bucal corto y redondeado; suturas notopleurales completas, basantra prosternal presente (Fig. 4B); pelta triangular; antenómeros III-IV con 2 y 4 conos sensoriales respectivamente (Fig. 4C) .................... Haplothrips

Haplothrips graminis Hood, única especie recolectada en ornamentales en Costa Rica (Retana-Salazar et al., 2014).

5b) Puente maxilar ausente, estiletes maxilares retraídos hasta el nivel de las sedas postoculares y muy cercanos entre sí (distancia entre ellos menor a un cuarto del ancho de la cabeza), cono bucal largo y puntiagudo extendiéndose más allá del prosterno, propleuron dividido en dos escleritos y con un par de sedas notopleurales, basantra prosternal ausente .............. Hoplandrothrips

Hoplandrothrips sp. Especie no identificada recolectada en Cuba (Suris \& González, 2008)

Este género incluye 17 especies registradas en la región Neotropical (Mound \& Marullo, 1996), muchas de ellas se alimentan de hongos de ramas caídas.

6a) Antena con nueve antenómeros, antenómeros III-IV con una o dos bandas de poros sensoriales paralelas al borde apical del segmento (Fig. 4D) ............... Heterothripidae Heterothrips

Heterothrips sericatus Hood y Heterothrips lankesteriensis Retana-Salazar se recolectaron con poca frecuencia en Costa Rica. La primera se encontró en flores de Citrus y de Mimosa sp. (Mound \& Marullo, 1996); mientras que adultos de la segunda especie, en flores de Malpighia glabra L. (Malpighiaceae), de importancia ornamental (Retana-Salazar, 2009).

6b) Antena con no más de ocho antenómeros, antenómeros III-IV con conos sensoriales simples bifurcados (Fig. $4 \mathrm{E}) \ldots \ldots \ldots \ldots \ldots \ldots$ Thripidae 7 7a) Cabeza, protórax (Fig. 4F, G) y patas fuertemente reticulados; endofurca mesotorácica y metatorácica, transversales, sin espínula; primera vena del ala anterior más o menos fusionada a la costa . . . . Panchaetothripinae 8 7b) Cabeza, protórax y patas con ornamentación estriada; endofurca mesotorácica en forma de U, con o sin espínula; primera vena del ala bien desarrollada y separada de la costa .................. Thripinae 11 
8a) Alas anteriores anchas, con la superficie reticulada y sedas mayores aplanadas, con aspecto de plumas por el raquis medio ..................Parthenothrips

Parthenothrips dracaenae (Heeger). Se asocia a Dracaena (Rusaceae) donde causa daños al follaje con lo que provoca pérdidas económicas. Gagné \& Montgomery (1985) informaron que grandes colonias de esta especie atacan el follaje de Dianella sp. (Xanthorrhoeaceae) en Hawai.

8b) Alas anteriores de dimensiones normales, nunca reticuladas y las sedas sin aspecto de plumas . . . . . . . 9 9a) Endofurca metatorácica en forma de lira (Fig. 5A) pero los brazos no tienden a tocarse en la línea media; conos sensoriales de los antenómeros III-IV bifurcados, antenómeros distales no forman un estilo acicular; pronoto rectangular; extremo distal del ala puntiagudo, sedas de las venas de las alas anteriores evidentes . . . . . . . . 10 9b) Endofurca metatorácica transversal, no se proyecta hacia el mesotórax; conos sensoriales de los antenómeros III-IV simples; antenómero distal acicular (Fig. 4F); sedas de las venas de las alas anteriores? muy reducidas; extremo distal del ala redondeado; pronoto con lados paralelos y ligeramente cóncavos........... Heliothrips

Heliothrips haemorrhoidalis Bouché, recolectada en toda el área de estudio de plantas de cítricos hasta helechos y en ornamentales de invernaderos (A.P. Retana-Salazar, com. pers). Esta especie es común en los invernaderos de Europa en particular en la Península Ibérica (Lacasa \& Llorens, 1996).

10a) Venas de las alas anteriores con hileras completas de sedas, fuertes y largas (Fig. 2C); cabeza constreñida cerca de la base dando la apariencia de un cuello; cabeza y pronoto con ornamentación fuerte y muy reticu-

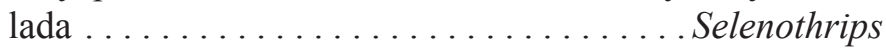

Selenothrips rubrocinctus (Giard), ampliamente distribuida y presente en toda el área de estudio. Se asocia al follaje de Terminalia catappa L. (Combretaceae) un árbol comercializado como ornamental. También se halla en las hojas del castaño de cajú (Anacardium occidentale L. (Anacardiaceae)) el cual se utiliza en algunas regiones como cerco vivo (Sordo \& Sordo, 2007) y es la única del género con valor económico (Prabhakaran Nair, 2010). También en follaje y frutos de cacao (Theobroma cacao L.).

10b) Primera vena del ala anterior con hilera de sedas dispuestas de forma irregular (Fig. 5B); cabeza no constreñida; cuerpo reticulado . . . . . . . . . . Caliothrips

Caliothrips phaseoli (Hood) ampliamente distribuida, se ha informado en Chrysanthemum coronarium L. (Amaranthaceae) en Cuba (Suris \& González, 2008)
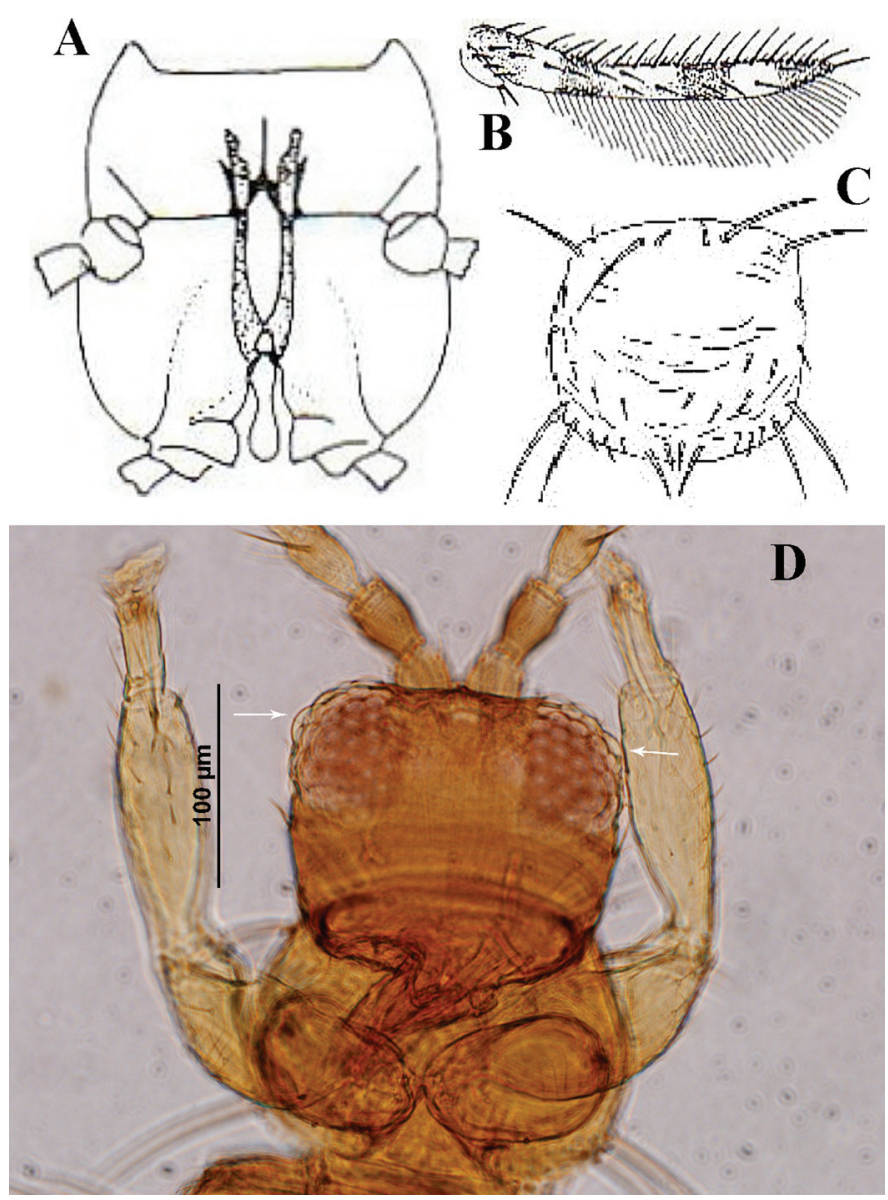

Figura 5. A. Dendrothrips sp., endofurca metatorácia con forma de lira (tomada de Mound et al., 1993). B. Caliothrips sp. Ala anterior (tomada de Mound et al., 1993). C. Pronoto típico de los géneros del grupo Frankliniella (tomada de Soto-Rodríguez \& Retana-Salazar, 2003). D. Exophthalmothrips fulvipennis (Moulton). Ojos mostrando los ommatidios laterales agrandados (foto Axel Retana-Salazar).

11a) Con ctenidia laterales a los espiráculos del tergo VIII ........................... 12 11b) Sin ctenidia laterales a los espiráculos del tergo VIII .......................... 15 12a) Ctenidia en posición anterior al espiráculo del tergo VIII; hileras de sedas sobre la primera y segunda venas del ala completas; generalmente con cuatro pares de sedas del pronoto bien desarrolladas (Fig.5C) . . . . . . . 13 12b) Ctenidia en posición posterior al espiráculo del tergo VIII; hileras de sedas sobre la primera y segunda venas interrumpidas o irregulares................ 14 13a) Omatidios laterales muy desarrollados, 1,5-2,0 veces el diámetro de los demás omatidios (Fig. 5D); especies grandes y de color castaño oscuro ... Exophthalmothrips 
Exophthalmothrips fulvipennis (Moulton) recolectada con frecuencia en Costa Rica en Megaskepasma (Acanthaceae) y en invernaderos de Impatiens sp. (Balsaminaceae) (Retana-Salazar, 1998)

13b) Omatidios laterales normales, de diámetro subigual a los demás omatidios............. Frankliniella

Género muy diverso en la región Neotropical, varias especies se hallan asociadas a cultivos de ornamentales en invernadero. Entre las más comunes se encuentran dos especies polífagas: Frankliniella occidentalis (Pergande) y Frankliniella insularis (Franklin). Esta última de alta prevalencia en Costa Rica, en algunas épocas del año; presenta más de 60 plantas hospedadoras registradas (Retana-Salazar \& Rodríguez-Arrieta, 2012). También ha sido informada en la Argentina asociada a algunos cultivos de importancia como ornamentales al igual que $F$. schultzei y F. gemina (Carrizo et al., 2008).

14a) Pronoto con tres-cuatro pares de sedas posteromarginales menores y con un par de sedas posteroangulares bien desarrolladas (Fig. 6A); antena con 7-8 antenómeros, margen posterior del tergo VIII con peine de desarrollo variable; esternos con o sin sedas accesorias (discales); primera vena del ala anterior con una hilera de sedas generalmente interrumpida................ Thrips

Tres especies se hallan en ornamentales, dos en $\mathrm{Cu}-$ ba, Thrips orientalis Bagnall y Thrips palmi Karny (Suris \& González, 2008) y dos en Costa Rica Thrips florum y Thrips tabaci (Retana-Salazar et al., 2014).

14b) Pronoto con cinco-seis pares de sedas posteromarginales menores muy pequeñas, sedas anteroangulares $\mathrm{y}$ posteroangulares de tamaño reducido (Fig. 6B); antena con 7 antenómeros, con craspeda de dientes triangulares y fuertes en el margen de los tergos (Fig. 6C); esternos con sedas accesorias (discales); hileras de sedas del ala anterior interrumpidas ...........Microcephalothrips

Microcephalothrips abdominalis (D. L. Crawford), única especie en el género asociada a varios cultivos ornamentales de Asteraceae en invernaderos (Mound \& Marullo, 1996).

15a) Apodema del pronoto presente; borde anterior del metaesterno redondeado (Fig. 6D); hileras de sedas ininterrumpidas en las venas de las alas anterio-

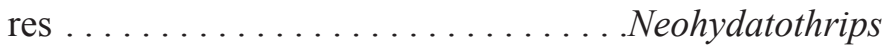

Neohydatothrips humberto Mound \& Marullo, asociada a malezas de los alrededores de invernaderos de ornamentales (Retana-Salazar et al., 2014).

$15 \mathrm{~b})$ Apodema del pronoto ausente; metaesterno variable en forma; venas de las alas anteriores en hileras de sedas completas ....................... 16 16a) Sensores en los antenómeros III-IV simples (bifurcados sólo en la especie asperatus); ala anterior con sedas de ápices expandidos (Fig. 6E); cabeza, pronoto y metanoto fuertemente reticulados; espínula mesotorácica bien

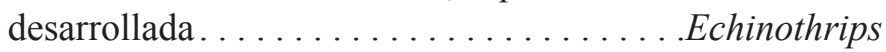

Echinothrips americanus Morgan. Es plaga del cultivo de Euphorbia pulcherrima Will. Ex Klotzsch (Euphorbiaceae), como en los cultivos de las Araceae Dieffenbachia y Syngonium, los cuales son valiosos por su follaje. Se informó también como plaga en Europa y en Tailandia, donde se registra más de 40 plantas hospedadoras, la mayoría son de importancia económica (Mound, 2009).

16b) Sensores de los antenómeros III-IV bifurcados (Fig.
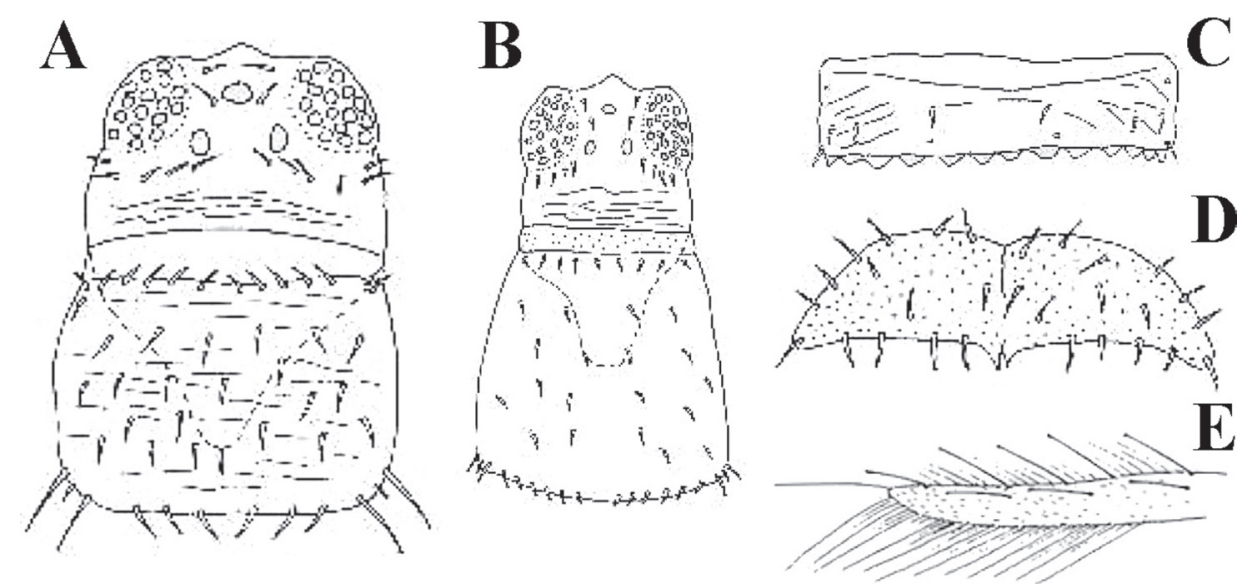

Figura 6. A. Cabeza y pronoto típico del género Thrips (tomada de Soto-Rodríguez \& Retana-Salazar, 2003). B. Cabeza y pronoto de Microcephalothrips. C. Tergo con craspedum típico del género Microcephalothrips (tomadas de Soto-Rodríguez \& Retana-Salazar, 2003). D. Neohydatothrips. Metaesterno (tomada de Mound et al., 1993). E. Echinothrips, ápice del ala anterior (tomada de Mound et al., 1993). 
7A, C); cabeza, pronoto y metanoto no reticulados (Fig. 8A); espínula mesotorácica presente o ausente . . . . 17 17a) Región de especialización alrededor del espiráculo en el tergo VIII muy reducida (Fig. 8B); pronoto con cinco pares de sedas posteromarginales; peine del margen posterior del tergo VIII ausente medialmente y con pocos dientes en las secciones laterales (Fig. 8B); seda apical del ala dos veces la longitud de la penúltima seda costal; tergo II abdominal sin sedas laterales; tercio anterior de los tergos ornamentados esculturados; (Fig. 8C). ......................... Gonzalezya

Gonzalezya marinae Retana-Salazar única especie endémica de Costa Rica asociada a Anthurium (RetanaSalazar, 2007).

17b) Región especializada alrededor de los espiráculos en el tergo VIII generalmente bien desarrollada (Fig. 9A,B), pronoto con cinco pares de sedas posteromarginales (incluyendo las angulares), peine en el margen posterior del tergo VIII ausente medialmente al menos en algunas es- pecies, seda costal anteroapical del ala I corta, nunca dos veces la longitud de la seda penúltima seda costal, tergo II abdominal con 3 pares de sedas marginales laterales, ornamentación del tercio anterior de los tergos abdominales variable ................. Chaetanaphothrips

Dos especies $C$. orchidii Moulton y $C$. signipennis Bagnall informadas en Costa Rica en orquídeas y Anthurium.

\section{DISCUSIÓN}

En este trabajo se recopila la información acerca de los principales géneros y especies de thrips asociados a los cultivos de ornamentales que es uno de los principales rubros de producción de exportación de la región, en especial en Costa Rica. La clave presentada se halla actualizada con los últimos cambios taxonómicos y responde a la revisión de material de diversas colecciones de América
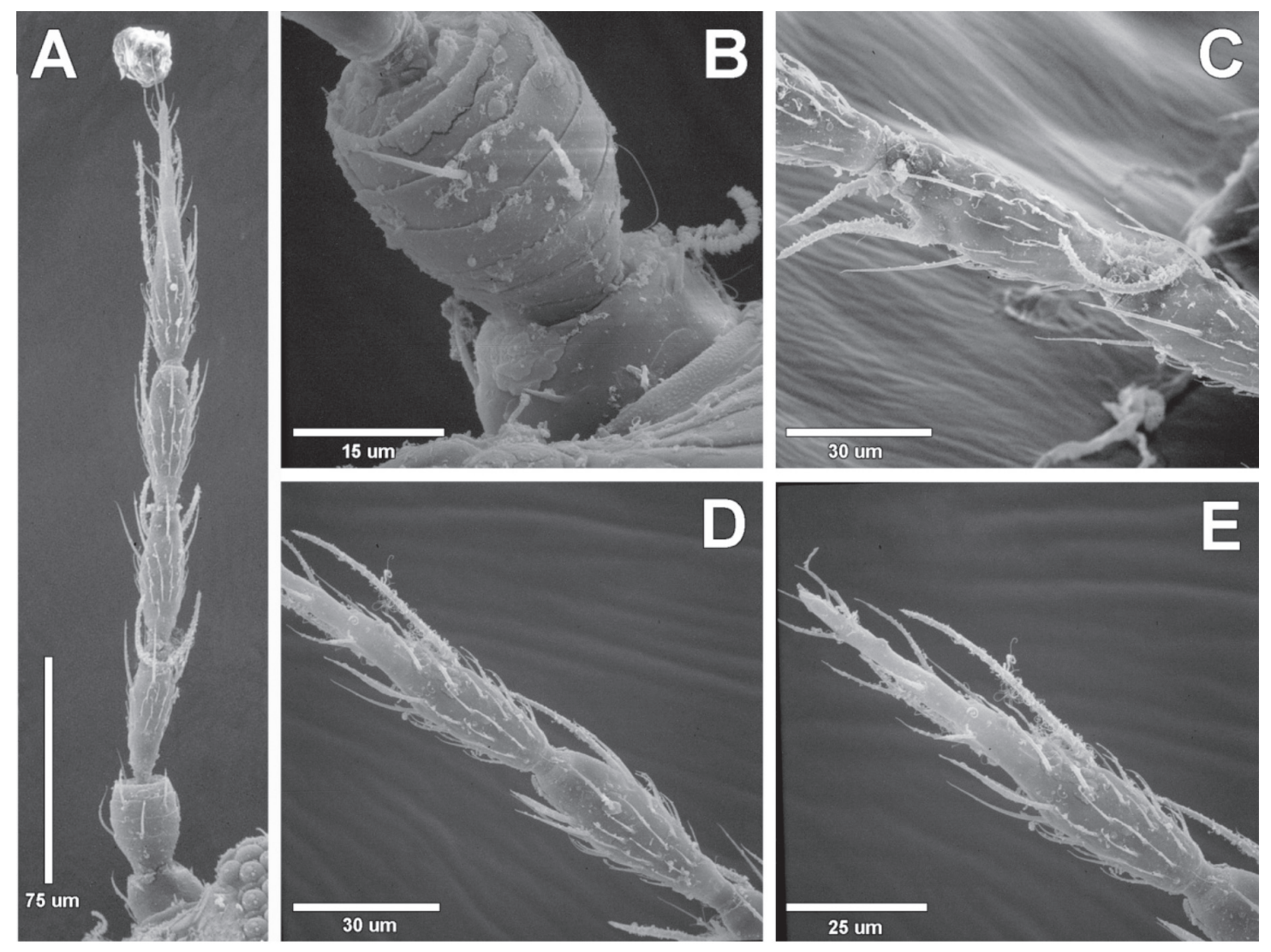

Figura 7. Detalles de la antena de Gonzalezya marinae Retana-Salazar. A. Vista general. B. Antenómeros I-II. C. Sensores en los antenómeros III-IV. D. Antenómeros V-VI. E. Estilo de la antena formado por los segmentos VI-VIII (fotografías SEM, tomadas de Retana-Salazar \& SotoRodríguez, 2013). 

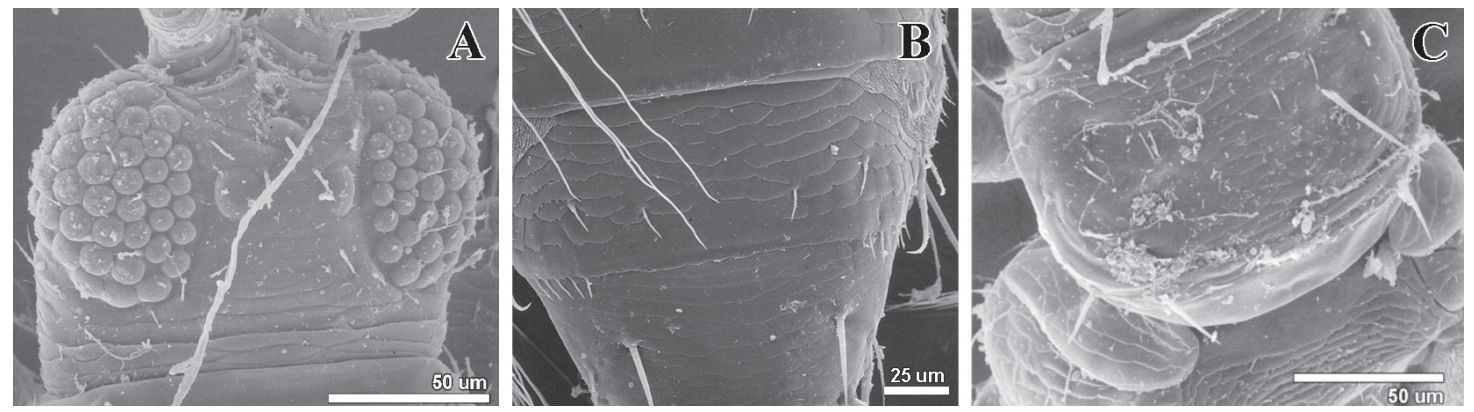

Figura 8. Gonzalezya marinae Retana-Salazar. A. Cabeza sin ornamentación reticular (Fotografías SEM, tomada de Retana-Salazar \& SotoRodríguez, 2013). B. Tergo abdominal VIII mostrando el área de especialización reducida y el peine interrumpido. C. Detalle del pronoto (fotografias SEM, tomadas de Retana-Salazar \& Soto-Rodríguez, 2013).
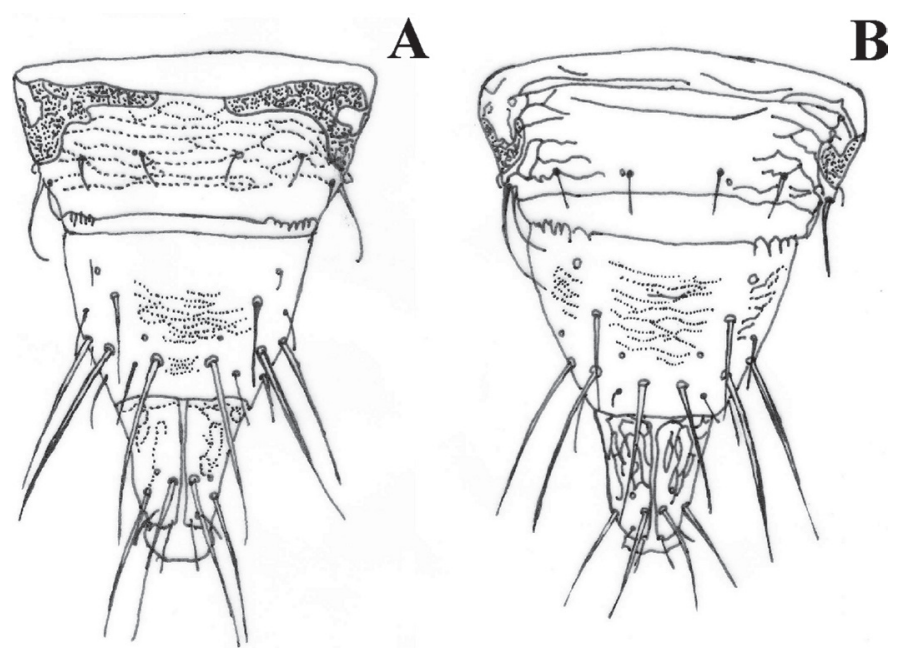

Figura 9. Chaetanaphothrips, dos especies C. orchidii Moulton y C. signipennis Bagnall informadas en Costa Rica en orquídeas y Anthurium. Tergos caudales en vista dorsal. Se muestran las áreas de especialización alrededor de los espiráculos en el tergo VIII (redibujadas de Nonaka \& Okajima, 1992).

Latina que han permitido generar un trabajo recopilatorio que, si bien es de mayor utilidad en América Central, puede ser utilizado en diferentes regiones de América Tropical.

AGRADECIMIENTOS. A la Vicerrectoría de Investigación de la Universidad de Costa Rica por el apoyo presupuestario para el desarrollo del proyecto "Caracterización morfológica y genética de adultos e inmaduros de especies de thrips (Insecta: Thysanoptera) asociadas a cultivos de plantas ornamentales en Costa Rica" 810-B2-A48, al Departamento de Parasitología Agraria de la Universidad Autónoma de Nayarit (UNA), México, a la Facultad de Agronomía, Universidad Agraria de la Habana (UNAH), Cuba; al Instituto de Biología de la Altura, Universidad Nacional (Jujuy, Argentina) por el apoyo en la ela- boración de este trabajo. A la Colección de Thysanoptera del Instituto de Biología, Universidad Nacional Autónoma de México (IBUNAM), México, al Museo de Senckenberg (SMF), Frankfurt, Alemania y al British Museum of Natural History (BMNH), Entomology, London, U.K por el préstamo de material. Al señor Carlos Martínez, por la colaboración en el dibujo científico de algunas de las imágenes del texto.

\section{LITERATURA CITADA}

Áreas-Sevilla, C. F. \& González-López, L. S. (2008). Estudio de la composición florística y sanidad forestal de la arboleda del sector sur del campus principal de la Universidad Nacional Agraria, Managua. Trabajo de Diploma. Universidad Nacional Agraria, Facultad de Agronomía, Departamento de Protección Agrícola y Forestal. 66 p.

Bhatti, J. S. (1971). A new Chaetanaphothrips-like genus form South India, with a redefinition of Chaetanaphothrips. Oriental Insects 5, 337-343.

Carrizo P, Gastelú C, Longoni P \& Klasman R. (2008). Especies de trips (Insecta: Thysanoptera: Thripidae) en las flores de ornamentales. IDESIA, 26, 83-86.

European Commission Directorate-General for Agriculture and Rural Development. (2006). WORKING DOCUMENT of The Commission staff on the situation of the flowers and ornamental plants sector, $40 \mathrm{p}$.

Federal Ministry of Food, Agriculture and Consumer Protection. 2010. Ornamental Plants Made in Germany, 4 p.

Gagné, W. C. \& Montgomery, S. L. (1985). Notes and exhibitions. Parthenothrips dracenae (Heeger). Proceedings Hawaiian Entomological Society 25, 9.

Hodges, A. W. \& Haydu, J. J. (2006). Economic impacts of the Florida environmental horticulture industry, 2000. EI 02-3, University of Florida, Institute of Food \& Agricultural Sciences, Department of Food \& Resource Economics, Gainesville. (http://www.fnga. org/documents/ImpactExecSummary.doc and www.FNGA.org.)

Jagadish, A. \& Ananthakrishnan, T. N. (1972). Taxonomic significance of the second instar larvae of some Indian Terebrantia (Thysanoptera: Insecta). Occasional Publications of the Entomo- 
logy Research Unit, 1. Entomology Research Unit, Loyola College, Madras.

Kondrashov, A. S. \& Kondrashov, F. A. (1999). Interactions among quantitative traits in the course of sympatric speciation. Nature 400, 351-354.

Lacasa-Plasencia, A. \& Llorens-Climent, J. M. (1996). Thrips y su control biológico (I). Serie Divulgativa Técnica 17. Edición Especial para la Consejería del Medio Ambiente, Agricultura y Agua de la Región de Murcia, 218 p.

Lewis, T. (1973). Thrips, their biology, ecology and economic importance. London, Academic Press, 349 p.

Mizell R., Knox G., Knight P., Gilliam C., Arthers S., Austin R., Baldwin H., Batson D., Boyd D. Jr., Bolques A., Braman K., Coker C., Chen Y., Cuevas P., Cuevas M., Czarnota M, DeMott J, Dobbs J, Donnan V, Fain G, Fare D, Faver M, Friday T, Fulcher A, Funderburk J, Gmeiner S, Hackney G, Hagan A, Hale F, Hayes J, Hendrix J, Horng J, Klingemen W, Gu M, Halcomb M, Hudson W, Humphries R, Kinard C, Kirker G, Ludwig S, Marshall J, May F, McLaurin W, Miller P, Mitchell T, Niu G, Norcini J, Oliver J, Posadas B, Rechcigl N, Rinehart T, Roebecker C, Schultz P, Simpson B, Story R, Tilt K, Webb S, Webb R \& Whitley K. (2010). Woody Ornamental and Landscape Plant Production and Pest Management Innovation Strategic Plan. 64 p.

Morris, D. C., Mound L. A., Schwarz M. P. \& Crespi B. J. 1999. Morphological Phylogenetics of Australian Gall-inducing Thrips and their Allies: The Evolution of Host-Plant Affiliations, Domicile Use, and Social Behaviour. Systematic Entomology 24, 289299.

Mound, L. A. (2009). National Diagnostic Protocol for Poinsettia Thrips, Echniothrips americanus. Department of Agriculture, Fisheries and Forestry, Government of Australia. 16 p. (reviewed 2012).

Mound, L. A., Retana-Salazar, A. P. \& du Heaume, G. J. (1993). Claves ilustradas para las familias y géneros de Terebrantia (Insecta: Thysanoptera) de Costa Rica y Panamá. Revista de Biología Tropical, 41, 709-727.

Mound, L. A. \& Marullo, R. (1996). The thrips of Central and South America: An Introduction (Insecta: Thysanoptera). Mem. Entomol., 487 p.

Nonaka, T. \& Okajima, S. (1992). Description of seven new species of the genus Chaetanaphothrips Priesner (Thysanoptera, Thripidae) from East Asia. Japan Journal of. Entomolugy, 60, 433-447.

Palmer, J. M. \& Mound, L. A. (1991). Thysanoptera. Chapter 22. 5: 67-76. In: Rosen, D. (Ed.), The Armoured Scale Insects, Their Biology, Natural Enemies and Control, Vol B. Amsterdam

Palmer, J. M, Mound, L. A. \& du Heaume, G. J. (1989). Thysanoptera 2. CIE Guides to Insects of Importance to Man. CAB International Institute of Entomology, British Museum Natural History, $73 \mathrm{p}$.
Prabhakaran Nair, K. P. (2010). The Agronomy and Economy of Important Tree Crops of the Developing World. London, UK, Elsevier, $313 \mathrm{p}$.

Programa de Certificación Fitosanitaria de Ornamentales de Exportación (PCFOE). (2008). Ministerio de Agricultura, Ganadería, Acuacultura y Pesca. Acuerdo 390. Registro Oficial de aprobación Programa de Certificación Fitosanitaria de Ornamentales de Exportación (PCFOE), Año II, Quito, Ecuador 48 p.

Retana-Salazar, A. P. (1998). Reestablecimiento de los géneros Frankliniella, Exophtalmothrips y Bolbothrips (Thysanoptera: Thripidae). Revista de Biología Tropical, 46, 385-396.

Retana-Salazar, A. P. (2007). Los tisanópteros del grupo genérico Anaphothrips (Thysanoptera: Thripidae), con énfasis en América Central. Revista de Biología Tropical, 55, 321-333.

Retana-Salazar, A. P. (2009). Species of Heterothrips Hood (Terebrantia: Heterothripidae) from Central America. Ceiba, 50, 10-17.

Retana-Salazar, A. P. \& Rodríguez-Arrieta, J. A. (2012). Aspectos de la biología de Frankliniella insularis Franklin 1908 (Thysanoptera: Thripidae) con especial énfasis en el sitio de pupación en la flor de Tabebuia rosea (Bertol) en el Valle Central de Costa Rica. Revista gaditana de Entomología 3, 69-84.

Retana-Salazar, A. P. \& Sánchez-Chacón, E. (2009). Anatomía de la agalla en Ficus benjamina (Moraceae) asociada a "thrips" (Tubulifera: Phlaeothripidae). Revista de Biología Tropical 57, 179186 (Supl).

Retana-Salazar, A. P. \& Soto-Rodríguez, G. A. (2013). Caracterización del grupo genérico Chaetanaphothrips Priesner en la Región del Neotrópico con énfasis en Gonzalezya (Terebrantia: Thripidae). Métodos en Ecología y Sistemática 8, 49-63, Volumen Especial.

Rodríguez-Arrieta, J. A. \& Retana-Salazar, A. P. (2010). Ultrastructure variability of Gynaikothrips uzeli-ficorum (Thysanoptera: Phlaeothripidae) complex in Ficus benjamina from Mexico and Costa Rica. Brenesia 73-74, 89-97.

Retana-Salazar, A. P., Rodríguez-Arrieta, J. A. \& González-Arce, M. E. (2014). Thrips (Thysanoptera) de los alrededores de invernaderos de ornamentales en Costa Rica, con notas sobre las formas inmaduras. Revista gaditana de Entomología, 5, 53-66.

Sordo, L \& Sordo, V. (2007). Especies utilizadas como cercas vivas por los productores en la Agricultura Urbana. O.B. ACTAF, Instituto de Investigaciones Forestales 1: 28-29.

Soto-Rodríguez, G. A. (2011). Diversidad de Thrips (Thysanoptera) en Costa Rica. Métodos en Ecología y Sistemática, 6(3), 34-43.

Soto-Rodríguez, G. A. \& Retana-Salazar, A. P. (2003). Clave ilustrada para los géneros de Thysanoptera y especies de Frankliniella presentes en cuatro zonas hortícolas en Alajuela, Costa Rica. Agronomía Costarricense, 27(2), 55-68.

Suris, M. \& González, C. (2008). Especies de trips asociadas a hospedantes de interés en las Provincias Habaneras. I. Plantas ornamentales. Revista de Protección Vegetal, 23(2), 80-84. 